\title{
CONVERGENT SUBSEQUENCES OF PARTIAL SUMS OF FOURIER SERIES OF $\varphi(L)$
}

\author{
S. V. KONYAGIN \\ Department of Mechanics and Mathematics, Moscow State University, 119992 Moscow, Russia \\ E-mail: konyagin@ok.ru
}

Let $\mathbb{T}=\mathbb{R} / 2 \pi \mathbb{Z}, L(\mathbb{T})$ be the set of all integrable functions $f: \mathbb{T} \rightarrow \mathbb{C}$. We associate with a function $f \in L(\mathbb{T})$ its trigonometric Fourier series

$$
f \sim \sum_{k=-\infty}^{\infty} \hat{f}(k) e^{i k x}, \quad \hat{f}(k)=\frac{1}{2 \pi} \int_{\mathbb{T}} f(x) e^{-i k x} d x .
$$

For $n \in \mathbb{N}$ define the $n$-th partial sum of $f$ as

$$
S_{n}(f ; x)=\sum_{k=-n}^{n} \hat{f}_{k} e^{i k x} .
$$

Let $\varphi:[0, \infty) \rightarrow[0, \infty)$ be a nonconstant convex function. Denote

$$
\varphi(L)=\left\{f \in L(\mathbb{T}): \int_{\mathbb{T}} \varphi(|f(x)|) d x<\infty\right\} .
$$

By $C_{1}, C_{2}, \ldots$ we denote absolute positive constants.

The paper is motivated by Ul'yanov's question: does there exist a sequence $\left\{N_{j}\right\}$ such that for every function $f \in L(\mathbb{T})$ there is an increasing sequence $\left\{n_{j}\right\}$ such that $n_{j} \leq N_{j}$ for all $j$ and $S_{n_{j}}(f) \rightarrow f$ almost everywhere? Note that existence of a nonrestricted sequence $\left\{n_{j}\right\}$ with almost everywhere convergence $S_{n_{j}}(f) \rightarrow f$ follows from the classical theorem of Kolmogorov $[\mathrm{K}]$. On the other hand, for any increasing sequence $\left\{n_{j}\right\}$ of positive integers there exists a real function $f \in L(\mathbb{T})$ such that $S_{n_{j}}(f)$ diverges almost everywhere $[\mathrm{G}]$ or even everywhere $[\mathrm{T}]$. Ul'yanov's problem is still open. However, for an Orlicz function space not coinciding with $L(\mathbb{T})$ a sequence $\left\{N_{j}\right\}$ depending on the space does exist.

Theorem 1. If $\varphi(u) / u \rightarrow \infty$ as $u \rightarrow \infty$ then there exists a sequence $\left\{N_{j}\right\}$ such that for every function $f \in \varphi(L)$ there is an increasing sequence $\left\{n_{j}\right\}$ such that $n_{j} \leq N_{j}$ for all $j$ and $S_{n_{j}}(f) \rightarrow f$ almost everywhere. 
Without loss of generality we can assume that

$$
\forall u \geq 0 \quad \phi(u) \geq u, \quad \int_{\mathbb{T}} \varphi(|f(x)|) d x \leq 1 .
$$

The main part of Theorem 1 is the following lemma.

Lemma 1. There exists a sequence $\left\{N_{j}\right\}(j \geq 0)$ such that for every $\varepsilon>0$ there is a sequence $\left\{n_{j}\right\}$ such that $N_{j-1}<n_{j} \leq N_{j}$ for all $j$ and $S_{n_{j}}(f) \rightarrow f$ on a complement to a subset of $\mathbb{T}$ of measure less than $\varepsilon$.

Theorem 1 easily follows from Lemma 1. Indeed, if $\left\{n_{j}\right\}=\left\{n_{j}\right\}(\varepsilon)$ is a sequence from the lemma, then there are $j(\varepsilon)$ and a set $E(\varepsilon) \in \mathbb{T}$ such that

$$
|E(\varepsilon)|<2 \varepsilon
$$

and

$$
\forall j \geq j(\varepsilon), x \in \mathbb{T} \backslash E(\varepsilon) \quad\left|f(x)-S_{n_{j}}(f ; x)\right| \leq \varepsilon .
$$

We can assume that $j\left(2^{-\nu-1}\right) \geq j\left(2^{-\nu}\right)$ for all $\nu \in \mathbb{N}$. Take $n_{j}$ arbitrary for $j<\nu(1)$, $n_{j}=n_{j}\left(2^{-\nu}\right)$ for $j\left(2^{-\nu}\right) \leq j<j\left(2^{-\nu-1}\right)$,

$$
E=\bigcap_{J} \bigcup_{j \geq J} E_{j}
$$

Then we have $|E|=0$ and $S_{n_{j}}(f ; x) \rightarrow f(x)$ for $x \in \mathbb{T} \backslash E$.

By $M f$ we denote Hardy-Littlewood's maximal function of $f$ :

$$
f(x)=\sup _{y<x<z} \frac{1}{z-y} \int_{y}^{z}|f(x)| d x .
$$

Let $M>0$ and

$$
E_{1}=\{x \in \mathbb{T}: M f(x)>M\} .
$$

Then $E_{1}$ is an open set. Note that, by (1),

$$
\int_{\mathbb{T}}|f(x)| d x \leq 1
$$

Using the weak-type $(1,1)$ inequality for $M f$ (see, for example, [D, p. 31]), we get $\left|E_{1}\right| \leq$ $2 / M$. We can write $E_{1}$ as a union of disjoint intervals

$$
E_{1}=\bigcup_{\mu}\left(y_{\mu}, z_{\mu}\right)
$$

Denote

$$
E_{2}=\bigcup_{\mu}\left(2 y_{\mu}-z_{\mu}, 2 z_{\mu}-y_{\mu}\right) .
$$

Then $\left|E_{2}\right| \leq 3\left|E_{1}\right| \leq 6 / M$. Hence, if $M \geq 7 / \varepsilon$, then

$$
\left|E_{2}\right|<\varepsilon \text {. }
$$

Our aim is to construct appropriate sequences $\left\{N_{j}\right\},\left\{n_{j}\right\}$ such that

$$
S_{n_{j}}(f ; x) \rightarrow f(x) \quad \text { almost everywhere on } \mathbb{T} \backslash E_{2} .
$$


We use the well-known Calderón-Zygmund decomposition of $f[\mathrm{CZ}]$. Let $g(x)=f(x)$ for $x \in \mathbb{T} \backslash E_{1}$ and

$$
g(x)=\frac{1}{z_{\mu}-y_{\mu}} \int_{y_{\mu}}^{z_{\mu}} f(x) d x
$$

for $x \in\left(y_{\mu}, z_{\mu}\right) \subset E_{1}$. It is easy to see that $|g(x)| \leq M$ almost everywhere. Indeed, if $x \in\left(y_{\mu}, z_{\mu}\right)$ and we assume $|g(x)|>M$, then

$$
\int_{y_{\mu}}^{z_{\mu}+\delta}|f(x)| d x>M\left(z_{\mu}+\delta-y_{\mu}\right)
$$

for some $\delta>0$, and hence $M f\left(z_{\mu}\right)>M$, but this is impossible. Further, almost everywhere on $\mathbb{T} \backslash E_{1}$ we have

$$
|g(x)|=|f(x)| \leq M f(x) \leq M .
$$

Therefore, since $g$ is essentially bounded, by Carleson's theorem [C] $S_{n}(g) \rightarrow g$ almost everywhere, and (5) is equivalent to

$$
S_{n_{j}}(f-g ; x) \rightarrow f(x)-g(x)=0 \quad \text { almost everywhere on } \quad \mathbb{T} \backslash E_{2} .
$$

By the way, we have proved that for any $\mu$ we have

$$
\int_{y_{\mu}}^{z_{\mu}}|g(x)| d x \leq \int_{y_{\mu}}^{z_{\mu}}|f(x)| d x \leq M\left|z_{\mu}-y_{\mu}\right| .
$$

Applying Jensen's inequality to a convex function $\varphi$ we get

$$
\int_{y_{\mu}}^{z_{\mu}} \varphi(|g(x)|) d x \leq \int_{y_{\mu}}^{z_{\mu}} \varphi(|f(x)|) d x .
$$

First, we construct sequences of positive numbers $\left\{L_{\nu}\right\} \rightarrow \infty$ and $\left\{\delta_{\nu}\right\} \rightarrow 0$. We take $L_{\nu} \geq 1$ so that

$$
\phi\left(L_{\nu}\right) / L_{\nu} \geq \nu
$$

Let $\delta_{1}=1 / 2$. If $\delta_{\nu}$ has been chosen, define

$$
\delta_{\nu+1}=\delta_{\nu}^{11} / L_{\nu}^{2} .
$$

We define $\left\{N_{j}\right\}$ to be

$$
N_{0}=1, \quad N_{j}=\left[1 / \delta_{j^{4}}\right](j \geq 1) .
$$

We may assume that $\varepsilon<1$. Since $\left|E_{2}\right|<\varepsilon$, the length of any interval $\left(y_{\mu}, z_{\mu}\right)$ is less than $1 / 3$. Now for any $\nu \geq 1$ we define

$$
\begin{aligned}
& f_{\nu}(x)= \begin{cases}f(x), & x \in\left(y_{\mu}, z_{\mu}\right), \delta_{\nu+1}<z_{\mu}-y_{\mu} \leq \delta_{\nu}, \\
0, & \text { otherwise },\end{cases} \\
& g_{\nu}(x)= \begin{cases}g(x), & x \in\left(y_{\mu}, z_{\mu}\right), \delta_{\nu+1}<z_{\mu}-y_{\mu} \leq \delta_{\nu}, \\
0, & \text { otherwise. }\end{cases}
\end{aligned}
$$

Clearly,

$$
f-g=\sum_{\nu}\left(f_{\nu}-g_{\nu}\right) .
$$


For any $j \geq 2$ we have, by (4),

$$
\sum_{\nu=(j-1)^{4}}^{j^{4}-1} \int_{\mathbb{T}}\left|f_{\nu}(x)\right| d x \leq \int_{\mathbb{T}}|f(x)| d x \leq 1 .
$$

Therefore, there exists $\nu_{0} \in\left[(j-1)^{4}, j^{4}\right)$ such that

$$
\int_{\mathbb{T}}\left|f_{\nu_{0}}(x)\right| d x \leq 1 /\left(j^{4}-(j-1)^{4}\right)<1 / j^{3} .
$$

By (7), also

$$
\int_{\mathbb{T}}\left|g_{\nu_{0}}(x)\right| d x<1 / j^{3}
$$

Denote

$$
h_{1}=\sum_{\nu<\nu_{0}}\left(f_{\nu}-g_{\nu}\right), \quad h_{2}=f_{\nu_{0}}-g_{\nu_{0}}, \quad h_{3}=\sum_{\nu>\nu_{0}}\left(f_{\nu}-g_{\nu}\right) .
$$

Identity (12) can be rewritten as

$$
f-g=h_{1}+h_{2}+h_{3} .
$$

By (13) and (14),

$$
\int_{\mathbb{T}}\left|h_{2}(x)\right| d x<2 / j^{3}
$$

Therefore, using $[\mathrm{K}]$, we obtain that for any $n$ there exists $F_{j, 2} \subset \mathbb{T}$ such that

$$
\left|F_{j, 2}\right| \leq 1 / j^{2}, \quad\left|S_{n}\left(h_{2} ; x\right)\right| \leq C_{1} / j\left(x \in \mathbb{T} \backslash F_{j, 2}\right) .
$$

Now, let us consider partial sums of the function $h_{1}$. We shall show that it is possible to choose $n_{j} \in\left(N_{j-1}, N_{j}\right]$ such that $\left|S_{n_{j}}\left(h_{1}\right)\right|$ will be small on a large subset of $\mathbb{T} \backslash E_{2}$. First, we deduce from (8) and convexity of $\varphi$ that

$$
\int_{\mathbb{T}} \varphi\left(\left|h_{1}(x) / 2\right|\right) d x \leq \frac{1}{2}\left(\int_{\mathbb{T}} \varphi(|f(x)|) d x+\int_{\mathbb{T}} \varphi(|g(x)|) d x\right) \leq \int_{\mathbb{T}} \varphi(|f(x)|) d x \leq 1 .
$$

Let

$$
h_{1}=h_{1,1}+h_{1,2},
$$

where $h_{1,1}(x)=h_{1}(x)$ for $\left|h_{1}(x)\right| \geq 2 L_{\nu_{0}}$ and $h_{1,1}(x)=0$ otherwise. We estimate partial Fourier sums of the function $h_{1,1}$ in the same way as for the function $h_{2}$. By (9) and (17),

$$
\begin{aligned}
\int_{\mathbb{T}}\left|h_{1,1}(x)\right| d x & =2 \int_{\mathbb{T}}\left|h_{1,1}(x) / 2\right| d x \leq \frac{2}{\nu_{0}} \int_{\mathbb{T}}\left|\varphi\left(h_{1,1}(x) / 2\right)\right| d x \\
& \leq \frac{2}{(j-1)^{4}} \int_{\mathbb{T}} \varphi\left(\left|h_{1}(x) / 2\right|\right) d x \leq \frac{32}{j^{4}} .
\end{aligned}
$$

Therefore, using $[\mathrm{K}]$ again, we obtain that for any $n$ there exists $F_{j, 1,1} \subset \mathbb{T}$ such that

$$
\left|F_{j, 1,1}\right| \leq 1 / j^{2}, \quad\left|S_{n}\left(h_{1,1} ; x\right)\right| \leq C_{2} / j^{2} \quad\left(x \in \mathbb{T} \backslash F_{j, 1,1}\right) .
$$

Now, let us estimate partial Fourier sums of the function $h_{1,2}$. Using (7) and (1) we have

$$
\int_{\mathbb{T}}\left|h_{1,2}(x)\right| d x \leq \int_{\mathbb{T}}|f(x)| d x+\int_{\mathbb{T}}|g(x)| d x \leq 2 \int_{\mathbb{T}}|f(x)| d x \leq 2 .
$$


Therefore,

$$
\int_{\mathbb{T}}\left|h_{1,2}(x)\right|^{2} d x \leq 4 L_{\nu_{0}}
$$

Fix $x \in \mathbb{T} \backslash E_{2}$. We use the well-known formula

$$
S_{n}\left(h_{1,2} ; x\right)=S_{n, 1}(x)+S_{n, 2}(x),
$$

where

$$
\begin{gathered}
S_{n, 1}(x)=\frac{1}{2 \pi} \int_{\mathbb{T}} h_{1,2}(t) \cot \left(\frac{t-x}{2}\right) \sin (n(t-x)) d t, \\
S_{n, 2}(x)=\frac{1}{2 \pi} \int_{\mathbb{T}} h_{1,2}(t) \cos (n(t-x)) d t .
\end{gathered}
$$

Now, observe that the supposition $h_{1,2}(t) \neq 0$ implies $t \in\left(y_{\mu}, z_{\mu}\right)$ for some $\mu$ with $z_{\mu}-y_{\mu} \geq \delta_{\nu_{0}}$. Also, since $x \in \mathbb{T} \backslash E_{2}$, we get $x \notin\left(2 y_{\mu}-z_{\mu}, 2 z_{\mu}-y_{\mu}\right)$. Thus,

$$
\left|\cot \left(\frac{t-x}{2}\right)\right| \leq \cot \left(\delta_{\nu_{0}} / 2\right) \text {, }
$$

and, by (20), we have

$$
\int_{\mathbb{T}}\left(h_{1,2}(t) \cot \left(\frac{t-x}{2}\right)\right)^{2} \leq C_{3} L_{\nu_{0}} /\left(\delta_{\nu_{0}}\right)^{2} .
$$

Now, we can use Parseval's identity

$$
\sum_{n}\left|S_{n, 1}(x)\right|^{2} \leq \frac{C_{3}}{4 \pi} L_{\nu_{0}} /\left(\delta_{\nu_{0}}\right)^{2} .
$$

For $x \in \mathbb{T} \backslash E_{2}$ denote

$$
\mathcal{N}_{1}(x)=\left\{n:\left|S_{n, 1}(x)\right|>\delta_{\nu_{0}}\right\}
$$

By (22), we have

$$
\left|\mathcal{N}_{1}(x)\right| \leq \frac{C_{3}}{4 \pi} L_{\nu_{0}} /\left(\delta_{\nu_{0}}\right)^{4}
$$

By integration we get

$$
\int_{\mathbb{T} \backslash E_{2}}\left|\mathcal{N}_{1}(x)\right| d x \leq C_{3} L_{\nu_{0}} /\left(\delta_{\nu_{0}}\right)^{4} .
$$

Similarly, if we denote

$$
\mathcal{N}_{2}(x)=\left\{n:\left|S_{n, 2}(x)\right|>\delta_{\nu_{0}}\right\}
$$

then

$$
\int_{\mathbb{T} \backslash E_{2}}\left|\mathcal{N}_{2}(x)\right| d x \leq C_{3} L_{\nu_{0}} /\left(\delta_{\nu_{0}}\right)^{4} .
$$

Denote

$$
N_{j}^{\prime}=N_{j-1}+\left[L_{\nu_{0}} /\left(\delta_{\nu_{0}}\right)^{5}\right]+1 .
$$

It follows from (23) and (24) that there exists $n, N_{j-1}<n \leq N_{j}^{\prime}$ such that

$$
\left|F_{j, 1,2}\right| \leq 2 C_{3} \delta_{\nu_{0}}<2 C_{3} / N_{j-1}
$$

where

$$
F_{j, 1,2}=F_{j, 1,2}(n)=\left\{x \in \mathbb{T} \backslash E_{2}: n \in \mathcal{N}_{1}(x) \cup N_{2}(x)\right\}
$$


By the definition of $\delta_{\nu_{0}+1}$ and $N_{j}$ we have $n<\left[1 / \delta_{\nu_{0}+1}\right] \leq N_{j}$, and we can take $n_{j}=n$. So, by (21), we have

$$
\forall x \in \mathbb{T} \backslash E_{2} \backslash F_{j, 1,2} \quad\left|S_{n_{j}}\left(h_{1,2} ; x\right)\right| \leq 2 \delta_{\nu_{0}}<2 / N_{j-1} .
$$

Now we will prove that for $n \leq N^{\prime}$ the partial sums $S_{n}\left(h_{3}\right)$ are uniformly small. Using (7), for any $k \in \mathbb{Z}$ and any $\mu$ we have

$$
\begin{aligned}
\left|\int_{y_{\mu}}^{z_{\mu}}(f(x)-g(x)) e^{-i k x} d x\right| & =\left|\int_{y_{\mu}}^{z_{\mu}}(f(x)-g(x))\left(e^{-i k x}-e^{-i k y_{\mu}}\right) d x\right| \\
& \leq|k|\left|z_{\mu}-y_{\mu}\right| \int_{y_{\mu}}^{z_{\mu}}|f(x)-g(x)| d x \leq 2|k|\left|z_{\mu}-y_{\mu}\right|^{2} M .
\end{aligned}
$$

Therefore,

$$
\begin{aligned}
\left|\hat{h}_{3}(k)\right| & =\left|\frac{1}{2 \pi} \sum_{z_{\mu}-y_{\mu} \leq \delta_{\nu_{0}+1}} \int_{y_{\mu}}^{z_{\mu}}(f(x)-g(x)) d x\right| \leq \frac{1}{2 \pi} \sum_{z_{\mu}-y_{\mu} \leq \delta_{\nu_{0}+1}} 2|k|\left|z_{\mu}-y_{\mu}\right|^{2} M \\
& \leq \frac{1}{\pi} \delta_{\nu_{0}+1}|k| M \sum_{z_{\mu}-y_{\mu} \leq \delta_{\nu_{0}+1}}\left|z_{\mu}-y_{\mu}\right| \leq 2 \delta_{\nu_{0}+1}|k| M
\end{aligned}
$$

and thus for any positive integer $n$

$$
\left|S_{n}\left(h_{3} ; x\right)\right| \leq \sum_{|k| \leq n}\left|\hat{h}_{3}(k)\right| \leq 4 \delta_{\nu_{0}+1} n^{2} M .
$$

In particular,

$$
\left|S_{n_{j}}\left(h_{3} ; x\right)\right| \leq 4 \delta_{\nu_{0}+1}\left(N_{j}^{\prime}\right)^{2} M
$$

It follows from the definition that $N_{j}^{\prime} \leq C_{4} L_{\nu_{0}} /\left(\delta_{\nu_{0}}\right)^{5}$. Consequently, by $(10), \delta_{\nu_{0}+1}\left(N_{j}^{\prime}\right)^{2}$ $\leq\left(C_{4}\right)^{2} \delta_{\nu_{0}}$, and after combining the last inequality with $(27)$ we obtain

$$
\left|S_{n_{j}}\left(h_{3} ; x\right)\right| \leq C_{5} M \delta_{\nu_{0}} \leq C_{5} M / N_{j-1} .
$$

To finish the proof, we define

$$
F_{j}=F_{j, 1,1} \cup F_{j, 1,2} \cup F_{j, 2} .
$$

By (16), (19), and (25),

$$
\left|F_{j}\right| \leq 2 / j^{2}+2 C_{3} / N_{j-1}
$$

Taking into account that, by construction, $\sum_{j} 1 / N_{j}<\infty$, we get

$$
\sum_{j}\left|F_{j}\right|<\infty \text {. }
$$

Further, we combine (16), (19), (25), and (28) with (18) and (15). Thus, if $x \in \mathbb{T} \backslash E_{2} \backslash F_{j}$, then

$$
\left|S_{n_{j}}(f-g ; x)\right| \leq C_{1} / j+C_{2} / j^{2}+\left(C_{5} M+2\right) / N_{j-1} \rightarrow 0 \quad(j \rightarrow \infty) .
$$

By $(29)$ and $(30), S_{n_{j}}(f-g ; x) \rightarrow 0$ almost everywhere on $\mathbb{T} \backslash E_{2}$. This proves (6) and completes the proof of Lemma 1.

For the whole class $L(\mathbb{T})$ we can construct a sequence $\left\{N_{j}\right\}$ with a weaker property than in Ul'yanov's problem. 
THEOREM 2. There exists a sequence $\left\{N_{j}\right\}$ such that for every function $f \in L(\mathbb{T})$ there is an increasing sequence $\left\{n_{j}\right\}$ such that $n_{j} \leq N_{j}$ for infinitely many $j$ and $S_{n_{j}}(f) \rightarrow f$ almost everywhere.

Without loss of generality we can assume that

$$
\int_{\mathbb{T}}|f(x)| d x \leq 1
$$

The following lemma is the main part of Theorem 2 .

Lemma 2. There exists a sequence $\left\{N_{j}\right\}(j \geq 0)$ such that for every $\varepsilon>0$ there is $S=S(\varepsilon)$ and also for sufficiently large $j$ there are numbers $N_{j-1}<n_{1}<\cdots<n_{j} \leq N_{j}$ such that

$$
\max \left(\left|S_{n_{1}}(f ; x)-f(x)\right|, \ldots,\left|S_{n_{j}}(f ; x)-f(x)\right|\right) \leq S
$$

on a complement to a subset of $\mathbb{T}$ of measure less than $\varepsilon$.

Theorem 2 follows easily from Lemma 2 . Indeed, let

$$
\varepsilon_{\mu}=2^{-\mu}, \quad \delta_{\mu}=\varepsilon_{\mu} / S\left(\varepsilon_{\mu}\right) \quad(\mu \geq 1) .
$$

For every $\mu$ there exists a trigonometric polynomial $P_{\mu}$ of degree $m_{\mu}$ such that

$$
\int_{\mathbb{T}}\left|f(x)-P_{\mu}(x)\right| d x \leq \delta_{\mu}
$$

Denote $g_{\mu}=f-P_{\mu}$. By Lemma 2 , for any $\mu$ there exist $j(\mu), E_{\mu} \subset \mathbb{T}, n_{1}(\mu), \ldots, n_{j(\mu)}(\mu)$ such that $j(\mu)>j(\mu-1)$ for $\mu>1, N_{j(\mu)-1}>m_{\mu},\left|E_{\mu}\right|<\varepsilon_{\mu}, N_{j(\mu)-1}<n_{1}(\mu)<\cdots<$ $n_{j(\mu)}(\mu) \leq N_{j(\mu)}$, and

$$
\max _{j}\left|S_{n_{j}(\mu)}\left(g_{\mu} ; x\right)-g_{\mu}(x)\right| \leq \varepsilon_{\mu}
$$

for $x \in \mathbb{T} \backslash E_{\mu}$. Since $f-g_{\mu}$ is a trigonometric polynomial of degree less than $n_{j}(\mu)$ for $j=1, \ldots, j(\mu),(33)$ can be rewritten as

$$
\max _{j}\left|S_{n_{j}(\mu)}(f ; x)-f(x)\right| \leq \varepsilon_{\mu} .
$$

We define a sequence $\left\{n_{j}\right\}$ to be the union of the sets $\left\{n_{1}(\mu), \ldots, n_{j(\mu)}(\mu)\right\}$ over $j \geq 1$. Define

$$
E=\bigcap_{J} \bigcup_{\mu \geq J} E_{\mu} .
$$

Then $n_{j(\mu)} \leq N_{j(\mu)}$ for all $\mu,|E|=0$, and $S_{n_{j}}(f ; x) \rightarrow f(x)$ for all $x \in \mathbb{T} \backslash E$.

In the proof of Lemma 2 we may assume that $\varepsilon<1$. We define $E_{1}, E_{2}, g$ as in the proof of Lemma 1. We consider that $M>18 / \varepsilon$. Then

$$
\left|E_{2}\right|<\varepsilon / 3 \text {. }
$$

It is enough to prove the existence of appropriate $S, n_{1}, \ldots, n_{j}$ such that

$$
\max \left(\left|S_{n_{1}}(f-g ; x)\right|, \ldots,\left|S_{n_{j}}(f-g ; x)\right|\right) \leq S
$$

everywhere on $\mathbb{T} \backslash E$, where

$$
E_{2} \subset E, \quad|E|<\varepsilon \text {. }
$$


A sequence $\left\{\delta_{j}\right\}$ will be constructed in the following way. Let $\delta_{1}=1 / 2$. If $\delta_{\nu}$ has been chosen, we consider the set $\mathcal{K}$ of continuous functions $h: \mathbb{T} \rightarrow \mathbb{C}$ such that for all $x, y \in \mathbb{T}$

$$
|h(x)| \leq 1 / \delta_{\nu}, \quad|h(x)-h(y)| \leq|x-y| / \delta_{\nu}^{2} .
$$

$\mathcal{K}$ is a compact subset of $C(\mathbb{T})$. Hence, there is a finite 1 -net $\left\{h_{1}, \ldots, h_{L_{\nu}}\right\}$ for $\mathcal{K}$ (that is, for any $h \in \mathcal{K}$ there is $l \leq L_{\nu}$ such that $\left.\left\|h-h_{l}\right\|_{C(\mathbb{T})} \leq 1\right)$. Observe that an 1-net with the same cardinality $L_{\nu}$ exists if the function class is defined on some compact subset of $\mathbb{T}$, since every function can be extended from the subset to $\mathbb{T}$ without change of the uniform norm and the Lipschitzian constant. Define

$$
\delta_{\nu+1}=\left(1 / \delta_{\nu}+\nu L_{\nu}^{2}\right)^{-2} .
$$

We define $\left\{N_{j}\right\}$ to be

$$
N_{0}=1, \quad N_{j}=1 / \delta_{j^{4}}(j \geq 1) .
$$

(Observe that $1 / \delta_{\nu}$ is an integer for any $\nu$.)

We define $f_{\nu}, g_{\nu}$, choose $\nu_{0} \in\left[(j-1)^{4}, j^{4}\right)$ for any $j \geq 2$ and further define $h_{1}, h_{2}, h_{3}$ as in the proof of Lemma 1 . We will seek for $n_{1}, \ldots, n_{j}$ from the segment $\left(1 / \delta_{\nu_{0}}, N_{j}^{\prime}\right.$ ] where

$$
N_{j}^{\prime}=1 / \delta_{\nu_{0}}+\nu_{0} L_{\nu_{0}}^{2}=\left(\delta_{\nu_{0}+1}\right)^{-1 / 2} .
$$

Similarly to (16) we prove that for some set $F_{j, 2}$ we have

$$
\left|F_{j, 2}\right| \leq 1 / j, \quad \max \left(\left|S_{n_{1}}\left(h_{2} ; x\right)\right|, \ldots,\left|S_{n_{j}}\left(h_{2} ; x\right)\right|\right) \leq C_{1} / j\left(x \in \mathbb{T} \backslash F_{j, 2}\right),
$$

Similarly to (27), we have a uniform estimate

$$
\max \left(\left|S_{n_{1}}\left(h_{3} ; x\right)\right|, \ldots,\left|S_{n_{j}}\left(h_{3} ; x\right)\right|\right) \leq 4 \delta_{\nu_{0}+1}\left(N_{j}^{\prime}\right)^{2} M=4 M .
$$

It suffices to estimate partial sums of the function $h_{1}$ on $\mathbb{T} \backslash E_{2}$.

Let us recall some well-known definitions and facts. For any function $h \in L(\mathbb{T})$ define the conjugate function

$$
\tilde{h}(x)=\frac{1}{2 \pi} \lim _{\delta \rightarrow 0} \int_{\delta \leq|t| \leq \pi} \frac{-h(x+t)}{\tan (t / 2)} d t .
$$

By the theorem of Lusin and Privalov (see, for example, [Z, 4.3 and 7.1]) this limit exists almost everywhere.

LEMma 2.1. There exists an absolute constant $C_{6}$ such that for any function $h \in L(\mathbb{T})$ and any $\alpha>0$

$$
\left|\left\{x:|\tilde{h}(x)|>\alpha \int_{\mathbb{T}}|h(t)| d t\right\}\right|<C_{6} / \alpha .
$$

This is the result of $[K]$.

For a positive integer $n$ define a modified Dirichlet kernel of order $n$ :

$$
D_{n}^{*}(t)=\frac{\sin (n t)}{\tan (t / 2)}
$$

LEMMA 2.2. Let

$$
S_{n}^{*}(h ; x)=\frac{1}{2 \pi} \int_{\mathbb{T}} D_{n}^{*}(t) h(x+t) d t .
$$


Then

$$
S_{n}^{*}(h ; x)=S_{n}(h ; x)-(\hat{h}(n) \exp (i n x)+\hat{h}(-n) \exp (-i n x)) / 2 .
$$

Therefore,

$$
\left|S_{n}(h ; x)\right| \leq\left|S_{n}^{*}(h ; x)\right|+\frac{1}{2 \pi} \int_{\mathbb{T}}|h(t)| d t .
$$

See the first statement in $[\mathrm{Z}, 2.3]$. The second statement follows immediately from the first one.

Denote $h^{(n)}(x)=h(x) e^{i n x}$.

LEMMA 2.3. We have

$$
S_{n}^{*}(h ; x)=i \exp (-i n x) \tilde{h}^{(n)}(x)-i \exp (i n x) \tilde{h}^{(-n)}(x)
$$

provided that the right-hand side of (39) is defined. Therefore,

$$
\left|S_{n}^{*}(h ; x)\right| \leq\left|\tilde{h}^{(n)}(x)\right|+\left|\tilde{h}^{(-n)}(x)\right| .
$$

This result is contained in $[\mathrm{Z}, 7.3]$.

We will use Lemmas 3-5 for functions $h^{(n)}, h=h_{1}$, and for $x \in \mathbb{T} \backslash E_{2}$. Note that for such $x$ and $h_{1}(x+t) \neq 0$ we have $|x-t| \geq \delta=\delta_{\nu_{0}}$. It is easy to see that

$$
|\cot (x / 2)| \leq 2 / \delta, \quad|\cot (x / 2)-\cot (y / 2)| \leq\left(2 / \delta^{2}+1\right)|x-y| \quad(|x|,|y| \in[\delta, \pi]) .
$$

Using also that, by (7) and (31),

$$
\int_{\mathbb{T}}\left|h_{1}(x)\right| d x \leq \int_{\mathbb{T}}|f(x)| d x+\int_{\mathbb{T}}|g(x)| d x \leq 2 \int_{\mathbb{T}}|f(x)| d x \leq 2,
$$

we have for any integer $n$ and $x, y \in \mathbb{T} \backslash E_{2}$

$$
\left|\tilde{h}_{1}^{(n)}(x)\right| \leq 1 / \delta, \quad\left|\tilde{h}_{1}^{(n)}(x)-\tilde{h}_{1}^{(n)}(y)\right| \leq|x-y| / \delta^{2} .
$$

Therefore, by the pigeon-hole principle, for $j \geq 3$ there exist $n_{1}, \ldots, n_{j}, 1 / \delta_{\nu_{0}}<n_{1}<$ $\cdots<n_{j} \leq N_{j}^{\prime}$, such that for all $x \in \mathbb{T} \backslash E_{2}$ and $\mu=2, \ldots, j$ we have

$$
\left|\tilde{h}_{1}^{\left(n_{\mu}\right)}(x)-\tilde{h}_{1}^{\left(n_{1}\right)}(x)\right| \leq 2, \quad\left|\tilde{h}_{1}^{\left(-n_{\mu}\right)}(x)-\tilde{h}_{1}^{\left(-n_{1}\right)}(x)\right| \leq 2 .
$$

(We have used that $\nu_{0} \geq j$ for $j \geq 3$.) Consequently, by Lemmas 2.2 and 2.3, and (40), we get

$$
\max \left(\left|S_{n_{1}}\left(h_{1} ; x\right)\right|, \ldots,\left|S_{n_{j}}\left(h_{1} ; x\right)\right|\right) \leq\left|\tilde{h}_{1}^{\left(n_{1}\right)}(x)\right|+\left|\tilde{h}_{1}^{\left(-n_{1}\right)}(x)\right|+5 .
$$

By Lemma 2.1, there is a set $F_{j, 1} \subset \mathbb{T}$ such that

$$
\left|F_{j, 1}\right|<\varepsilon / 3
$$

and for any $x \in \mathbb{T} \backslash F_{j, 1}$ we have

$$
\left|\tilde{h}_{1}^{\left(n_{1}\right)}(x)\right|+\left|\tilde{h}_{1}^{\left(-n_{1}\right)}(x)\right| \leq C_{7} / \varepsilon
$$

and thus, by (42), for $x \in \mathbb{T} \backslash E_{2} \backslash F_{j, 1}$

$$
\max \left(\left|S_{n_{1}}\left(h_{1} ; x\right)\right|, \ldots,\left|S_{n_{j}}\left(h_{1} ; x\right)\right|\right) \leq C_{7} / \varepsilon+5 .
$$

Denote

$$
E=F_{j, 1} \cup F_{j, 1} \cup E_{2} .
$$


By (34), (37), and (42), the conditions (36) are satisfied for sufficiently large $j$. Using (15), (37), (38), and (43), we have

$$
\max \left(\left|S_{n_{1}}(f-g ; x)\right|, \ldots,\left|S_{n_{j}}(f-g ; x)\right|\right) \leq C_{1} / j+4 M+C_{7} / \varepsilon+5
$$

for all $x \in \mathbb{T} \backslash E$. This proves (35) and completes the proof of Lemma 2 .

The author was supported by the grant 02-01-00248 from the Russian Foundation for Basic Research and the grant N NSh-3004.2003.1.

\section{References}

[C] L. Carleson, On convergence and growth of partial sums of Fourier series, Acta Math. 116 (1966), 133-157.

[CZ] A. P. Calderón and A. Zygmund, On the existence of certain singular integrals, Acta Math. 88 (1952), 85-139.

[D] J. Duoandikoetxea, Fourier Analysis, Amer. Math. Soc., Providence, RI, 2001.

[G] R. P. Gosselin, On the divergence of Fourier series, Proc. Amer. Math. Soc. 9 (1958), $278-282$.

[K] A. N. Kolmogoroff, Sur les fonctions harmoniques conjuguées et les séries de Fourier, Fund. Math. 7 (1925), 24-29.

[T] V. Totik, On the divergence of Fourier series, Publ. Math. (Debrecen) 29 (1982), 251-264.

[Z] A. Zygmund, Trigonometric Series, Vol. 1, Cambridge Univ. Press, Cambridge, 1977. 